\title{
Raising the awareness for a possible COVID-19 postinfectious neurocognitive syndrome
}

\author{
Walter Sena \\ Department of Psychiatry, Federal University of Rio de Janeiro, Brasil
}

\begin{abstract}
Neurologic and sensory symptoms of COVID-19 are common, including olfactory dysfunction, headache, and dizziness. Other betacoronaviruses show neuroinvasiveness properties, and there is evidence that it may be partially responsible for the acute respiratory failure induced by SARS-CoV-2. Sudden onset anosmia is a prevalent symptom in a large number of paucisymptomatic patients and is probably caused by a dysregulation of the olfactory neuroepithelium. Early signs of anosmia can predict memory impairments even among cognitively healthy individuals. Hence, it is essential to study neurocognition in patients with a history of anosmia and COVID-19.
\end{abstract}

SARS-CoV-2 | COVID-19 | neuroinvasion | anosmia | postinfection | neurocognition

Correspondence: walter.sena@ufrj.br

\section{Introduction}

After the pandemic is over, Coronavirus Disease 19 (COVID19) will continue as epidemic waves until a vaccine is widely available. One of the possible long-term consequences is a postinfectious neurocognitive syndrome. The first report of acute neurologic symptoms found that 78 out of 214 patients had central nervous symptoms, as headache, dizziness, impaired consciousness, ataxia, and convulsion (1). SARSCoV-2 RNA was detected in a patient cerebral spinal fluid (2) and similar findings were described during the SARS-CoV-1 epidemic, such as samples of autopsies with viral genome sequences confined to the cytoplasm of neurons (3).



Fig. 1. Possible route of neuroinvasion

\section{Neuroinvasion and Neuropathogenesis}

There is evidence that the potential neuroinvasiveness of SARS-CoV-2 is partially responsible for the acute respiratory failure of COVID-19 (4). Neuroinvasion has been documented for other betacoronaviruses, including Severe Acute Respiratory Syndrome Coronavirus (SARS-CoV-1), Middle East Respiratory Syndrome Coronavirus (MERS$\mathrm{CoV})$, Human Coronavirus ( $\mathrm{HCoV})$, Mouse Hepatitis Virus (MHV) and Porcine Hemagglutinating Encephalomyelitis Virus (PHEV) (5-9).

A viral flora in the brain can induce neuro-immunopathology in susceptible individuals or direct neuronal damage (10). For instance, $\mathrm{HCoV}$ RNA was found in the brain parenchyma of infeceted patients (11). Furthermore, SARS-CoV-2 may conceal itself inside neurons from the immune recognition, what means that complete clearance of the virus may not be guaranteed even after recovery from acute infection (4). 


\section{Neurologic and sensory symptoms}

Neurologic symptoms can present both as central nervous system symptoms, such as headache, dizziness, impaired consciousness, ataxia, and seizures, and as sensory symptoms, such as anosmia/hyposmia and ageusia/dysgeusia (1). Also, the possible dysfunction of the cardiorespiratory center could be another central nervous system symptom (4) since SARS-CoV-2 could have invaded it via a transynaptic route from mechano- and chemoreceptors in the respiratory tract $(12,13)$.

Sudden onset anosmia with or without dysgeusia is a prevalent sensory symptom in a large number of paucisymptomatic patients $(14,15)$ and is probably caused by a dysregulation of non-neuronal cells in the olfactory neuroepithelium that coexpress SARS-CoV-2 receptor and entry genes (16).

There are no descriptions yet of chronic neurologic symptoms caused by SARS-CoV-2, but researches have already described other CoVs postinfectious neurologic manifestations. A study from Saudi Arabia showed brain lesions after infection with MERS-CoV in three patients (17). In this study, brain MRI revealed widespread bilateral hyperintense lesions on T2-weighted imaging within the white matter and subcortical areas of the frontal, temporal, and parietal lobes, the basal ganglia, and corpus callosum.

Other viral infections, such as Measles and Rubella, can cause a slowly progressive neurological disease that presents several years after the acute viral illness has occurred. Human $\mathrm{T}$ cell lymphotropic virus type 1 (HTLV-1) can cause slowly progressive myelopathy in adulthood with no prior history of apparent infection. Human herpes viruses, herpes simplex virus and varicella-zoster virus, are the prototypical viruses that establish lifelong latent infections in human peripheral and CNS sensory ganglia that may episodically reactivate, resulting in acute infection (18).

\section{Neurocognitive symptoms}

There is an increased risk of cognitive deficits in viral postinfectious syndromes (19). Although extensively underreported, infection is the most common cause of encephalitis and viruses are the most prevalent etiological agents (20).

Each virus cause specific postinfectious neurocognitive syndromes that will depend on early diagnosis and treatment, age and the cognitive level at the onset, and site and extent of neuronal injury. Japanese Encephalitis (JE), for instance, has an estimated 50,000 cases and 15,000 deaths annually, and about half of the survivors have severe neuropsychiatric sequelae (21). West Nile Fever and JE link to significant long-term neurocognitive deficits, including decreases in psychomotor speed and in verbal and visuospatial learning, which not only persist but also worsen for years after recovery (22). Other viruses may also cause long-term lower scores on tests of fine motor and psychomotor skills as well as on learning and memory (23).

SARS-CoV-2 causes a local dysregulation of the nasal neuroepithelium that may open a path through the cribriform plate to olfactory bulb. From there the virus may reach the entorhinal cortex via the olfactory tract, and later the hippocampus, which, if affected, will impair memory. Accordingly, early signs of anosmia can predict memory impairments even among cognitively healthy individuals (24).

\section{Future perspectives}

Scientists should raise the awareness for non-negligible longterm consequences of a possible COVID-19 postinfectious neurocognitive syndrome. It is not yet possible to ascertain that SARS-CoV-2 causes direct neuronal damage since only a few studies assessed neurological symptoms, and no studies assessed neurocognitive symptoms this far. Hence, it is essential to systematically study cognition in patients with a history of anosmia and COVID-19. Along this line, psy- 
chiatrists and psychologists when examining these patients should require a neurocognitive assessment. Finally, considering the required physical distancing/isolation, a plan of action to develop a global standardized online neurocognitive assessment is foremost.

\section{Bibliography}

1. Ling Mao, Huijuan Jin, Mengdie Wang, Yu Hu, Shengcai Chen, Quanwei He, Jiang Chang, Candong Hong, Yifan Zhou, David Wang, Xiaoping Miao, Yanan Li, and Bo Hu. Neurologic Manifestations of Hospitalized Patients With Coronavirus Disease 2019 in Wuhan, China. JAMA Neurology, April 2020. ISSN 2168-6149. doi: 10.1001/jamaneurol.2020.1127.

2. Takeshi Moriguchi, Norikazu Harii, Junko Goto, Daiki Harada, Hisanori Sugawara, Junichi Takamino, Masateru Ueno, Hiroki Sakata, Kengo Kondo, Natsuhiko Myose, Atsuhito Nakao, Masayuki Takeda, Hirotaka Haro, Osamu Inoue, Katsue Suzuki-Inoue, Kayo Kubokawa, Shinji Ogihara, Tomoyuki Sasaki, Hiroyuki Kinouchi, Hiroyuki Kojin, Masami Ito, Hiroshi Onishi, Tatsuya Shimizu, Yu Sasaki, Nobuyuki Enomoto, Hiroshi Ishihara, Shiomi Furuya, Tomoko Yamamoto, and Shinji Shimada. A first Case of Meningitis/Encephalitis associated with SARS-Coronavirus-2. International Journal of Infectious Diseases, page S1201971220301958, April 2020. ISSN 12019712. doi: 10.1016/j.ijid.2020.03.062.

3. Jiang Gu, Encong Gong, Bo Zhang, Jie Zheng, Zifen Gao, Yanfeng Zhong, Wanzhong Zou, Jun Zhan, Shenglan Wang, Zhigang Xie, Hui Zhuang, Bingquan Wu, Haohao Zhong, Hongquan Shao, Weigang Fang, Dongshia Gao, Fei Pei, Xingwang Li, Zhongpin $\mathrm{He}$, Danzhen Xu, Xeying Shi, Virginia M. Anderson, and Anthony S.-Y. Leong. Multiple organ infection and the pathogenesis of SARS. Journal of Experimental Medicine, 202(3): 415-424, August 2005. ISSN 1540-9538, 0022-1007. doi: 10.1084/jem.20050828.

4. Yan-Chao Li, Wan-Zhu Bai, and Tsutomu Hashikawa. The neuroinvasive potential of SARSCoV2 may be at least partially responsible for the respiratory failure of COVID-19 patients. Journal of Medical Virology, page jmv.25728, March 2020. ISSN 0146-6615, 1096-9071. doi: $10.1002 /$ jmv. 25728 .

5. William G. Glass, Kanta Subbarao, Brian Murphy, and Philip M. Murphy. Mechanisms of Host Defense following Severe Acute Respiratory Syndrome-Coronavirus (SARS-CoV) Pulmonary Infection of Mice. The Journal of Immunology, 173(6):4030-4039, September 2004. ISSN 0022-1767, 1550-6606. doi: 10.4049/jimmunol.173.6.4030.

6. Kun Li, Christine Wohlford-Lenane, Stanley Perlman, Jincun Zhao, Alexander K. Jewell, Leah R. Reznikov, Katherine N. Gibson-Corley, David K. Meyerholz, and Paul B. McCray. Middle East Respiratory Syndrome Coronavirus Causes Multiple Organ Damage and Lethal Disease in Mice Transgenic for Human Dipeptidyl Peptidase 4. Journal of Infectious Diseases, 213(5):712-722, March 2016. ISSN 0022-1899, 1537-6613. doi: 10.1093/infdis/jiv499.

7. Mathieu Dubé, Alain Le Coupanec, Alan H. M. Wong, James M. Rini, Marc Desforges, and Pierre J. Talbot. Axonal Transport Enables Neuron-to-Neuron Propagation of Human Coronavirus OC43. Journal of Virology, 92(17):e00404-18, /jvi/92/17/e00404-18.atom, June 2018. ISSN 0022-538X, 1098-5514. doi: 10.1128/JVI.00404-18.

8. Xinying Zhou, Fen Huang, Lei Xu, Zhanmin Lin, Femke M. S. de Vrij, Ane C. Ayo-Martin, Mark van der Kroeg, Manzhi Zhao, Yuebang Yin, Wenshi Wang, Wanlu Cao, Yijin Wang, Steven A. Kushner, Jean Marie Peron, Laurent Alric, Robert A. de Man, Bart C. Jacobs, Jeroen J. van Eijk, Eleonora M. A. Aronica, Dave Sprengers, Herold J. Metselaar, Chris I. de Zeeuw, Harry R. Dalton, Nassim Kamar, Maikel P. Peppelenbosch, and Qiuwei Pan. Hepatitis E Virus Infects Neurons and Brains. The Journal of Infectious Diseases, 215(8): 1197-1206, April 2017. ISSN 0022-1899, 1537-6613. doi: 10.1093/infdis/jix079.

9. Zi Li, Wenqi He, Yungang Lan, Kui Zhao, Xiaoling Lv, Huijun Lu, Ning Ding, Jing Zhang, Junchao Shi, Changjian Shan, and Feng Gao. The evidence of porcine hemagglutinat- ing encephalomyelitis virus induced nonsuppurative encephalitis as the cause of death in piglets. PeerJ, 4:e2443, September 2016. ISSN 2167-8359. doi: 10.7717/peerj.2443.

10. Marc Desforges, Alain Le Coupanec, Philippe Dubeau, Andréanne Bourgouin, Louise Lajoie, Mathieu Dubé, and Pierre J. Talbot. Human Coronaviruses and Other Respiratory Viruses: Underestimated Opportunistic Pathogens of the Central Nervous System? Viruses, 12(1):14, December 2019. ISSN 1999-4915. doi: 10.3390/v12010014.

11. N. Arbour, R. Day, J. Newcombe, and P. J. Talbot. Neuroinvasion by Human Respiratory Coronaviruses. Journal of Virology, 74(19):8913-8921, October 2000. ISSN 0022-538X. doi: 10.1128/JVI.74.19.8913-8921.2000.

12. Selma Hadziefendic and Musa A Haxhiu. CNS innervation of vagal preganglionic neurons controlling peripheral airways: a transneuronal labeling study using pseudorabies virus. Journal of the Autonomic Nervous System, 76(2-3):135-145, May 1999. ISSN 01651838. doi: 10.1016/S0165-1838(99)00020-X.

13. Madhu Kalia and M-Marsel Mesulam. Brain stem projections of sensory and motor components of the vagus complex in the cat: II. Laryngeal, tracheobronchial, pulmonary, cardiac, and gastrointestinal branches. The Journal of Comparative Neurology, 193(2):467-508, September 1980. ISSN 0021-9967, 1096-9861. doi: 10.1002/cne.901930211.

14. Jerome R. Lechien, Carlos M. Chiesa-Estomba, Daniele R. De Siati, Mihaela Horoi, Serge D. Le Bon, Alexandra Rodriguez, Didier Dequanter, Serge Blecic, Fahd El Afia, Lea Distinguin, Younes Chekkoury-Idrissi, Stéphane Hans, Irene Lopez Delgado, Christian Calvo-Henriquez, Philippe Lavigne, Chiara Falanga, Maria Rosaria Barillari, Giovanni Cammaroto, Mohamad Khalife, Pierre Leich, Christel Souchay, Camelia Rossi, Fabrice Journe, Julien Hsieh, Myriam Edjlali, Robert Carlier, Laurence Ris, Andrea Lovato, Cosimo De Filippis, Frederique Coppee, Nicolas Fakhry, Tareck Ayad, and Sven Saussez. Olfactory and gustatory dysfunctions as a clinical presentation of mild-tomoderate forms of the coronavirus disease (COVID-19): a multicenter European study. European Archives of Oto-Rhino-Laryngology, April 2020. ISSN 0937-4477, 1434-4726. doi: 10.1007/s00405-020-05965-1.

15. Andrea Giacomelli, Laura Pezzati, Federico Conti, Dario Bernacchia, Matteo Siano, Letizia Oreni, Stefano Rusconi, Cristina Gervasoni, Anna Lisa Ridolfo, Giuliano Rizzardini, Spinello Antinori, and Massimo Galli. Self-reported olfactory and taste disorders in SARS-CoV-2 patients: a cross-sectional study. Clinical Infectious Diseases, page ciaa330, March 2020. ISSN 1058-4838, 1537-6591. doi: 10.1093/cid/ciaa330.

16. Leon Fodoulian, Joel Tuberosa, Daniel Rossier, Basile N. Landis, Alan Carleton, and Ivan Rodriguez. SARS-CoV-2 receptor and entry genes are expressed by sustentacular cells in the human olfactory neuroepithelium. preprint, Neuroscience, April 2020.

17. Y. M. Arabi, A. Harthi, J. Hussein, A. Bouchama, S. Johani, A. H. Hajeer, B. T. Saeed, A. Wahbi, A. Saedy, T. AIDabbagh, R. Okaili, M. Sadat, and H. Balkhy. Severe neurologic syndrome associated with Middle East respiratory syndrome corona virus (MERSCoV). Infection, 43(4):495-501, August 2015. ISSN 0300-8126, 1439-0973. doi: 10.1007/s15010-015-0720-y.

18. Karen L Roos. Viral Infections. In Christopher Goetz, editor, Textbook of Clinical Neurology, page 24. Saunders, Chicago, 3rd edition, 2007.

19. Julia Granerod, Nicholas W. S. Davies, Parashar P. Ramanuj, Ava Easton, David W. G. Brown, and Sara L. Thomas. Increased rates of sequelae post-encephalitis in individuals attending primary care practices in the United Kingdom: a population-based retrospective cohort study. Journal of Neurology, 264(2):407-415, February 2017. ISSN 0340-5354, 1432-1459. doi: 10.1007/s00415-016-8316-8.

20. Julia Granerod and Natasha S. Crowcroft. The epidemiology of acute encephalitis. Neuropsychological Rehabilitation, 17(4-5):406-428, August 2007. ISSN 0960-2011, 1464 0694. doi: 10.1080/09602010600989620.

21. T. Solomon. NEUROLOGICAL ASPECTS OF TROPICAL DISEASE: Japanese encephalitis. Journal of Neurology, Neurosurgery \& Psychiatry, 68(4):405-415, April 2000. ISSN 00223050. doi: 10.1136/jnnp.68.4.405.

22. Kristy O. Murray, Melissa N. Garcia, Mohammad H. Rahbar, Diana Martinez, Salma A. Khuwaja, Raouf R. Arafat, and Susan Rossmann. Survival Analysis, Long-Term Outcomes, and Percentage of Recovery up to 8 Years Post-Infection among the Houston West Nile 
Virus Cohort. PLoS ONE, 9(7):e102953, July 2014. ISSN 1932-6203. doi: 10.1371/journal. pone. 0102953 .

23. Else Quist-Paulsen, Vidar Ormaasen, Anne-Marte B. Kran, Oona Dunlop, Per Magne Ueland, Thor Ueland, Randi Eikeland, Pål Aukrust, and Tonje H. Nordenmark. Encephalitis and aseptic meningitis: short-term and long-term outcome, quality of life and neuropsychological functioning. Scientific Reports, 9(1):16158, December 2019. ISSN 2045-2322. doi: 10.1038/s41598-019-52570-2

24. Rosebud O. Roberts, Teresa J. H. Christianson, Walter K. Kremers, Michelle M. Mielke, Mary M. Machulda, Maria Vassilaki, Rabe E. Alhurani, Yonas E. Geda, David S. Knopman, and Ronald C. Petersen. Association Between Olfactory Dysfunction and Amnestic Mild Cognitive Impairment and Alzheimer Disease Dementia. JAMA Neurology, 73(1):93, January 2016. ISSN 2168-6149. doi: 10.1001/jamaneurol.2015.2952. 